\title{
Valentin L. Popov: Contact Mechanics and Friction: Physical Principles and Applications
}

\author{
Springer-Verlag, Berlin, Heidelberg, 2010, ISBN 978-3-642-10802-0, \\ e-ISBN 978-3-642-10803-7, DOI 10.1007/978-3-642-10803-7
}

Martin Müser

Received: 12 July 2010/Accepted: 15 July 2010/Published online: 25 July 2010

(C) Springer Science+Business Media, LLC 2010

\begin{abstract}
Although contact mechanics and friction has been studied for centuries, no definitive textbook has emerged on these topics. Part of the reason for this shortcoming may lie in the multi-disciplinary nature of tribology, which ideally requires a competent knowledge of the mechanics of continuous media, materials science, and various sub-disciplines of physics such as statistical mechanics. The largest difficulty in writing a textbook may be related to the absence of a generally applicable formalism, such as perturbation theory or linear response theory, with which one could tackle systematically tribological problems. Consequently, most treatises on the topic either have picture book quality or in the other extreme are so complicated that even the most educated and talented graduate students cannot follow. Valentin Popov has managed to find a compromise in his book Contact Mechanics and Friction. His treatise contains many explanations and model calculations, which capture the essence of the given problem and yet do not require complicated formalisms. In this way, Popov puts a reader equipped with good intuition into a position to tackle quite a few tribological problems in a semi-quantitative fashion.
\end{abstract}

The 20 chapters deal with a variety of engineering and physical aspects of tribology. Examples are microscopic models for the origin of friction or superlubricity, rolling contacts, rubbing-induced wear, rheology of lubricants, squeaking brakes, and earthquakes. The book is organized extremely well-in particular when it comes to Hertzian contact mechanics and its generalizations. It is regrettable, however, that Persson's contact mechanics theory and its consequences have not been discussed and instead preference was given to the Greenwood and Williamson theory, which is based on assumptions that are now disproved.

The large number of solved problems at the end of each chapter is a definite advantage of the book. This is why it can be of great benefit to those who teach tribology. Nonetheless, some effort has to be made in order to understand the finesse of various calculations. Meaningful references are provided for those who want to refine the material as a teacher or to indulge in tribological research. Overall, Contact Mechanics and Friction is a well-written if not desperately needed textbook on tribology. If more effort is made to improve on the didactics of the book in future editions, it may even become a standard treatise.

The original of this review will be published in German in the Physik Journal, August/September 2010.

M. Müser ( $\square)$

Universität des Saarlandes, Lehrstuhl für Materialsimulation,

Campus C6 3, 66123 Saarbrücken, Germany

e-mail: martin.mueser@mx.uni-saarland.de 